\title{
Fiber Density Estimation by Tensor Divergence
}

\author{
Marco Reisert, Henrik Skibbe, and Valerij G. Kiselev \\ Medical Physics, University Medical Center Freiburg, \\ Breisacher Str. 60a, 79106 Freiburg, Germany
}

\begin{abstract}
Diffusion-sensitized magnetic resonance imaging provides information about the fibrous structure of the human brain. However, this information is not sufficient to reconstruct the underlying fiber network, because the nature of diffusion provides only conditional fiber densities. That is, it is possible to infer the percentage of bundles that pass a voxel with a certain direction, but the absolute number of fibers is inaccessible. In this work we propose a conservation equation for tensor fields that can infer this number up to a factor. Simulations on synthetic phantoms show that the approach is able to derive the densities correctly for various configurations. In-vivo results on 20 healthy volunteers are plausible and consistent, while a rigorous evaluation is difficult, because conclusive data from both MRI and histology remain elusive even on the most studied brain structures.
\end{abstract}

\section{Introduction}

The representation of neuronal fibers is of major interest in neuroscience and medicine. Diffusion weighted magnetic resonance imaging (DWI) has the potential to visualize non-invasively these fiber [1]. The quantitative reconstruction of the underlying fiber architecture is a great challenge, as DWI gives only indirect measures for the presence of neuronal fibers. One of the main problems is that the ordinary DWI measurement can only provide relative information. For each voxel one can infer a probability distribution of directions (fiber or diffusion orientation distributions), but these distributions do not give information about the total number of fibers within the voxel. There are attempts to measure fiber density directly, e.g. by determining the myelinization degree with bound pool fraction imaging [2] or with optimized diffusion measurements and multicompartement models [3]. On the other hand, fiber tracking approaches can be used to infer tract-density maps [45], but the inferred maps are usually correlated closely with fractional anisotropy (FA) [6]. This work attempts to formulate a fiber conservation law, which is able to derive fiber density maps from ordinary (clinical suitable) DWI by employing the global coherence of the data. The idea is inspired by fluid dynamics, where mass conservation is expressed by a partial differential equation. A similar equation is formulated for tensor fields representing the underlying diffusion measurement. While in fluid dynamics the divergence equation can be derived from a strict integral formulation, our derivation remains 'empirical', but simulations show that the obtained solutions work well. The proposed equation is solved via a finite element method on a standard PC in a few minutes. 


\section{Method}

In fluid dynamics, the mass conservation is expressed by the equation $\partial_{i}\left(v_{i}(\boldsymbol{r}) \rho(\boldsymbol{r})\right)=$ 0 , where $\boldsymbol{v}=\left(v_{1}, v_{2}, v_{3}\right)$ and $\rho$ are the velocity field and the particle density at a point $\boldsymbol{r}=\left(r_{1}, r_{2}, r_{3}\right)$, respectively, and $\partial_{i}=\partial / \partial r_{i}$. (We use Einstein's summation convention throughout this paper, all indices appearing more than once are implicitly summed). In fluid flows each point $\boldsymbol{r}$ is assigned a unique velocity $\boldsymbol{v}(\boldsymbol{r})$. The case of fibers is different. At a finite resolution it is possible that several fibers with different directions cross a volume element. Detailed information about the fiber orientations is thus provided by the absolute joint fiber distribution $p(\boldsymbol{n}, \boldsymbol{r})$ that depends on the unit vector $n \in S_{2}$ defining the fiber direction and position $\boldsymbol{r}$. This function is not directly accessible with DWI. The DWI observable quantity is rather the conditional distribution of directions $p(\boldsymbol{n} \mid \boldsymbol{r})$, which does not provide any information about the absolute density of fibers $\rho(\boldsymbol{r})$. These quantities are related as $p(\boldsymbol{n}, \boldsymbol{r})=p(\boldsymbol{n} \mid \boldsymbol{r}) \rho(\boldsymbol{r})$ provided the normalization $\int_{S_{2}} p(\boldsymbol{n} \mid \boldsymbol{r}) d \boldsymbol{n}=1$. So, our goal is to is to find a 'fiber density' $\rho(\boldsymbol{r})$ for a given conditional orientation density $p(\boldsymbol{n} \mid \boldsymbol{r})$. However note, that we can infer $\rho$ only up to a global normalization factor, that is, we cannot infer an 'absolute' fiber density map. This problem is ill-posed, which can be illustrated with an example of isotropic $p(\boldsymbol{n} \mid \boldsymbol{r})$. Such a distribution can result from fibers with any density. We thus need some additional prior knowledge to regularize the problem. We seek for a cost functional that favors reasonable configurations. The cost functional is constructed by using the principle of fiber continuity [7], which assumes that fibers appear to be locally straight. The main idea is to require $n_{i} \partial_{i} p(\boldsymbol{n}, \boldsymbol{r})$ to be small in magnitude. The term $n_{i} \partial_{i} p(\boldsymbol{n}, \boldsymbol{r})$ is the change in the number of fibers with direction $\boldsymbol{n}$ when moving in direction $\boldsymbol{n}$. However, this assumption is too restrictive not being able to cope with curved fibers. Thus, we want to build costs that are more selective. Therefore, consider the vector field $n_{i} n_{j} \partial_{j} p(\boldsymbol{n}, \boldsymbol{r})$. This vector points in an arbitrary selected direction $\boldsymbol{n}$ and its magnitude equals the gradient of the fiber density in this direction. We sum this vector over all fiber directions to obtain an integral characteristics of a voxel:

$$
k_{i}(\boldsymbol{r})=\int_{S_{2}} n_{i} n_{j} \partial_{j} p(\boldsymbol{n}, \boldsymbol{r}) d \boldsymbol{n}=\partial_{j} \int_{S_{2}} n_{i} n_{j} p(\boldsymbol{n}, \boldsymbol{r}) d \boldsymbol{n}=\partial_{j} P_{i j}(\boldsymbol{r})
$$

Note that this vector field $\boldsymbol{k}=\left(k_{1}, k_{2}, k_{3}\right)$ only depends on the projection $P_{i j}(\boldsymbol{r})=$ $\int n_{i} n_{j} p(\boldsymbol{n}, \boldsymbol{r})$, which is an ordinary Cartesian rank-2 tensor. As we know that $p(\boldsymbol{n}, \boldsymbol{r})=$ $p(\boldsymbol{n} \mid \boldsymbol{r}) \rho(\boldsymbol{r})$, the tensor $P_{i j}$ can also be expressed in terms of the projection of the conditional $T_{i j}(\boldsymbol{r})=\int n_{i} n_{j} p(\boldsymbol{n} \mid \boldsymbol{r})$ as follows:

$$
P_{i j}(\boldsymbol{r})=\rho(\boldsymbol{r}) \int_{S_{2}} n_{i} n_{j} p(\boldsymbol{n} \mid \boldsymbol{r}) d \boldsymbol{n}=\rho(\boldsymbol{r}) T_{i j}(\boldsymbol{r}) .
$$

Considering the example of parallel fibers, it is clear that $\boldsymbol{k}=0$ expresses the fact that the fibers do not terminate. So, a non-zero $k$ has to be suppressed in this case. On the other hand, $k$ defines the curvature field for bending fiber: 1 and should not be penalized

\footnotetext{
${ }^{1}$ For simplicity we give a $2 \mathrm{D}$ example. The bending configuration is given by $\left(n_{1}^{\mathrm{fib}}, n_{2}^{\mathrm{fib}}\right)=$ $\left(-r_{2}, r_{1}\right) /|\boldsymbol{r}|$ from which we have $\left(P_{i j}\right)=\frac{1}{|\boldsymbol{r}|^{2}}\left(\begin{array}{cc}r_{2}^{2} & -r_{1} r_{2} \\ -r_{1} r_{2} & r_{1}^{2}\end{array}\right)$. Computing the partial derivatives we get $k_{j}=\partial_{i} P_{i j}=-\left(r_{1}, r_{2}\right) /|\boldsymbol{r}|^{2}$ and hence $|\boldsymbol{k}|=1 /|\boldsymbol{r}|$ which was to show.
} 
in that case. To implement such a selected suppression of $\boldsymbol{k}$, we use the fact that the curvature vector of a fiber is orthogonal to its tangent. Instead of looking for $\rho$ 's that minimize the squared norm of the field $\boldsymbol{k}$, we use the tensor $T_{i j}$ as a metric tensor to project out the pure curvature contributions. That is, we look for a fiber density that minimizes the cost functional

$$
J_{\mathrm{TD}}(\rho)=\int_{\Omega} k_{i} T_{i j} k_{j} d \boldsymbol{r}=\int_{\Omega}\left(\partial_{a} P_{i a}\right) T_{i j}\left(\partial_{b} P_{j b}\right) d \boldsymbol{r}
$$

We used $T_{i j}$ instead of $P_{i j}$ as metric tensor, because this keeps our objective quadratic in $\rho$, and hence, we can solve the problem by inverting a linear system. Note that for a constant isotropic field $T_{i j}=\delta_{i j}$ our equation reduces to the integral over the gradient magnitude of $\rho$ which is equivalent to Laplace's equation. Thus, more isotropic tensors $T_{i j}$ lead to more spatially homogeneous fiber densities.

\subsection{Boundary Conditions and Implementation via Finite Elements}

As the objective as given in equation (3) is of differential nature we have to keep care what happens at the boundary $\partial \Omega$ of the white matter domain. The weakest assumption is a Neumann boundary condition, that is $v_{j} \partial_{j} \rho(\boldsymbol{r})=0$ for $\boldsymbol{r} \in \partial \Omega$ and $\boldsymbol{v}$ orthogonal to the boundary. However, in this setting our minimization problem is solved by the trivial solution $\rho=0$, hence we need additional assumptions to keep the solution away from being zero. The simplest way is to set $\rho$ on the boundary (in the transition area of white and gray matter) to a specific value (Dirichlet condition). Unfortunately, we need an idea of the amount of fiber terminals to set $\rho$ to reasonable values. Of course, a uniform distribution is an option. Another way is to modify the objective. We figured out two ways: one can subtract the total number of fibers $J(\rho)=J_{\mathrm{TD}}(\rho)-\int_{\Omega} \rho$ such that the consistency term $J_{\mathrm{TD}}$ is minimized and simultaneously the total number of fibers $\int_{\Omega} \rho$ is maximized. Secondly, one can add a quadratic deviation from an initial guess $\rho_{0}$, that is $J(\rho)=J_{\mathrm{TD}}(\rho)+\lambda \int_{\Omega}\left|\rho-\rho_{0}\right|^{2}$. If $\rho_{0}=1$ and $\lambda$ sufficiently small, this approach converges towards the first option. We will discuss the different options in the experiments.

To solve the minimization problem we followed a finite element (FEM) discretization which is ideally suited for our problem. We cannot give here a complete introduction to FEM and refer to [8], but shortly propose which element we used and what is important during discretization. To stay as close as possible to the initial Cartesian discretization of the data we use a trilinear finite element, that is, the elements are cubes with corners in the center of each voxel, where the interpolation function within this cube is the ordinary trilinear one frequently used in computer graphics. Thus, the unknown variables are just the density values at the voxel positions. The advantage is that we can treat the tensor field $T_{i j}(\boldsymbol{r})$ in the same manner. The values of $T_{i j}$ are known at the voxel centers and the value within the finite element can also be computed by trilinear interpolation. Each unknown variable is associated with a basis function. The density $\rho$ is a linear combination of all basis functions $\rho(\boldsymbol{r})=\sum_{\beta} a_{\beta} \phi_{\beta}(\boldsymbol{r})$ where $\beta$ runs over all voxels, $a_{\beta}$ are the unknowns and $\phi_{\beta}$ are the basis functions. In FEM we have to compute for all pairs of basis function $\phi_{\mu}, \phi_{\beta}$ the integral $M_{\mu, \beta}=\int_{\Omega}\left(\partial_{a} T_{i a} \phi_{\mu}\right) T_{i j}\left(\partial_{b} T_{j b} \phi_{\beta}\right) d \boldsymbol{r}$, which forms the matrix which one has 
to inver2. For the computation of the integrals we used the MATLAB symbolic toolbox. Assembling the matrix with a standard desktop PC needs about 3 minutes for half a million unknowns. Solving the problem by an iterative method is in the same time range (we used the MATLAB minres algorithm with a tolerance of $10^{-5}$ ).

\section{Experiments}

We start with simulations on synthetic data to show that the solutions of our equations give quantitative results. Three configurations are considered: a fanning, a bending and a crossing. The fanning was generated by parabolas, where the opening degree varies with the $y$-axis section 3 . For the crossing we varied the angle and the relative density of the tracts. To generate the tensor field $T_{i j}$ we followed the approach described above, i.e. $T_{i j}=n_{i}^{\mathrm{fib}} n_{j}^{\mathrm{fib}}$. Of course, the field is always normalized such that $T_{i i}=1$. The configurations were rendered on a $128 \times 128 \times 3$ matrix. Finally, we smoothed the field $T_{i j}$ with a Gaussian of width 1 voxel. We investigated the three different boundary assumption discussed above: using Dirichlet boundaries in the regions where the fibers terminate (DIR), or modifying the objective by subtracting the total number of fibers (LIN), or adding the quadratic deviation from a uniform density $\rho_{0}=1$ (QUAD). In Figure 1 we show densities inferred with DIR/LIN/QUAD assumptions. The Dirichlet (DIR) boundaries are the 'easiest' case, as the most prior knowledge is put in. All three configurations are well resolved in this case. For the fanning we checked the fiber number conservation by integrating the density along the y-axis. Ideally, it should be a constant value. And, in fact, up to some fluctuation due to discretization it is a constant. During the narrowing the packing is getting dense, and during the widening the density decreases. The two crossing tracts (crossing angle of 60 degrees) are assumed to have a density ratio $2: 1$. For an orthogonal crossing this is expressed by the fact that the eigenvalues of $T_{i j}$ in the crossing region have also the ratio of $2: 1$. Also the DIR boundary values were set to this ratio. Obviously the crossing is nicely resolved, also quantitatively the values within the crossing regions are the sum of the values of the two inflowing tracts. Finally, the result for the bending is, as desired, a constant density. For the LIN approach the results are a bit different. For the crossing the results do not change, which is encouraging. Without putting any prior knowledge about the in- and out-flowing fibers the equation is able to infer the relative densities perfectly. For the bending case (Figure 1f),g)) we get different results, tracts with high curvature are penalized, although we have shown above that for a perfect bending $T_{i j} \partial_{j} P_{a j}=0$. That is, curved fibers should not be penalized. We found that this is basically a discretization artifact. The sharper the bending the higher the errors in the discretized computation of $T_{i j} \partial_{j} P_{a j}$. However, it is not astonishing that the solution is so sensitive. Theoretically,

\footnotetext{
${ }^{2}$ For the computation of $M_{\mu, \beta}$ there is an important note. To compute the product $T_{j b} \phi_{\beta}$ within an element one has to first multiply the values of $T_{j b}$ and $\phi_{\beta}$ at the element corners and then interpolate within the element. We found that it is not possible to interpolate both independently and then multiply the interpolants.

${ }^{3}$ The fanning is represented by a family of parabolas $y_{a}(x)=a\left(1+b x^{2}\right)$ where $a \in$ $(-d / 2, d / 2)$ and $d$ is the thickness of the bundle. The parameter $b$ controls the degree of fanning
} 


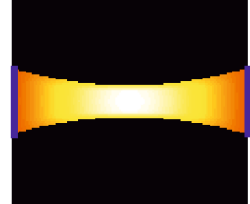

a) fanning $D I R$

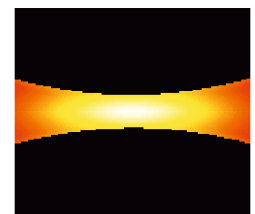

c) fanning LIN

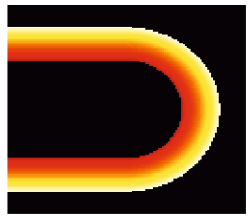

f) bending LIN

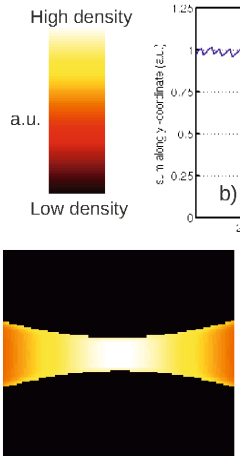

d) fanning QUAD

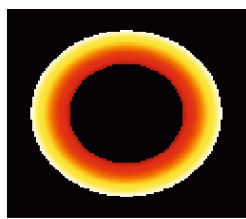

g) circle LIN
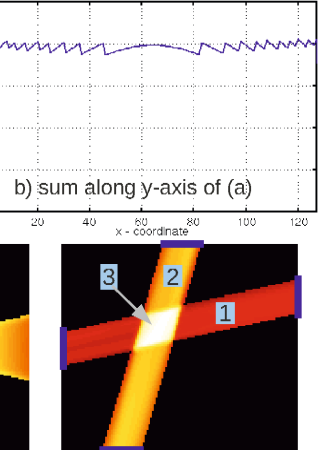

e) crossing DIR/LIN/QUAD

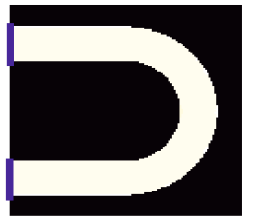

h) bending DIR/QUAD

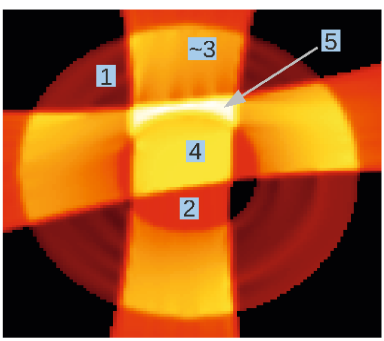

i) combination QUAD

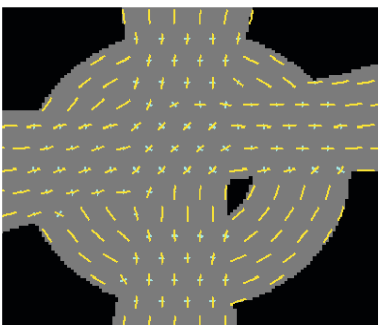

j) combination EVs of tensor field

Fig. 1. Fiber densities of the synthetic phantoms. The blue bars in a,e,h) indicate the Dirichlet boundaries. a) The fanning with DIR boundary. b) The sum along the y-axis of the densities in (a) to show the fiber number preservation. c) Fanning with LIN approach: the central, noncurved fibers are emphasized. d) For the QUAD approach there is nearly no difference to DIR. e) The crossing is well resolved for all three boundary approaches, the density values in the indicated regions have the desired ratio 1:2:3. f,g) The LIN approach tends to give lower density to curved fibers, which is a discretization artifact. $\mathbf{h}$ ) for the DIR and QUAD approach the density is constant over the bending. i) density of complex configuration with annotated ratios. $\mathbf{j}$ ) the eigenvectors of the tensor $T_{i j}$ representing the experimental data.

there is huge family of solutions to the LIN based equation. Any density profile is a valid solution as long as the profile do not change along the bundle. The situation is similar like in fluid dynamics: imagine a non-viscous compressible fluid flowing in a tube with constant velocity $\boldsymbol{v}=\left(v_{0}, 0,0\right)$. Any density profile $\rho(x, y, z)=\rho_{0}(y, z)$ is a solution to the equation $\partial_{i}\left(v_{i} \rho\right)=0$. The QUAD approach resolves this ambiguity, which can be seen in Figure 1h). Actually, the QUAD approach can be seen as a small Tikhonov-regularization with $\lambda \int \rho^{2}$ to make the equation less sensitive to the discretization artifact, because $\int|\rho-1|^{2}=\int \rho^{2}-2 \int \rho+$ const. We found a regularization parameter of $\lambda=1 / n$ to work well, where $n$ is the number of unknown variables. The discretization artefacts disappear and for all configurations (Figure 1 d,e,h) the densities stay quantitative, i.e. the densities of the crossing keep the assumed ratios, for the fanning the fiber number stays constant and for the bending we get a uniform density. In a further experiment (Figure 1,j) we found that the results also generalize: a combination of a fanning, crossing and bending. Each of the crossing tracts has a density of 2 at its most narrow location, while the 'circle' configuration has density 1. Up to some small discretization artifacts the densities are well inferred. We want to emphasize that although our approach soley relies on a tensor representation, the densities for the threefold crossing are predicted well. 


\subsection{In-Vivo Data}

To investigate our approach on real DTI data we considered 20 scans of healthy volunteers at a b-value of $1 \mathrm{~ms} / \mu \mathrm{m}^{2}$ with 61 diffusion directions and an isotropic resolution of $2 \mathrm{~mm}^{3}$. A white matter probability map was generated with SPM (Version 5, http://www.fil.ion.ucl.ac.uk/spm/) on a T1-weighted scan, which was co-registered to the $b_{0}$-scan of the diffusion sequence. The probability maps are thresholded at 0.1 to get the simulation domain $\Omega$. The diffusion weighted images were up-sampled to a $1 \mathrm{~mm}^{3}$-resolution. We decided to base the conditional distribution $p(\boldsymbol{n} \mid \boldsymbol{x})$ purely on the diffusion tensor, thus the following experiments are also appropriate in a clinical setting with low diffusion weighting. We used the formula $p(\boldsymbol{n} \mid \boldsymbol{x}) \propto e^{-n_{i} D_{i, j}^{-1} n_{j} r_{0}^{2} /(2 t)}$, where $D^{-1}$ is the inverted diffusion tensor, $r_{0}$ a distance and $t$ the diffusion time. That is, $p(\boldsymbol{n} \mid \boldsymbol{x})$ is the probability that a particle is displaced by an amount of $r_{0}$ in direction $\boldsymbol{n}$ within the time $t$. It is important to control the sharpness (entropy) of the distribution. A high entropy will lead to more uniform fiber densities than a low entropy. The parameter $r_{0}^{2} / t$ controls this sharpness. We found a value of $r_{0}^{2} / t=25 \mu \mathrm{m}^{2} / \mathrm{ms}$ appropriate. Of course, a more detailed investigation is needed at this point. The tensor $T_{i j}$ is computed via $T_{i j}(\boldsymbol{r})=\int n_{i} n_{j} p(\boldsymbol{n} \mid \boldsymbol{r})$ numerically and normalized such that $T_{i i}=1$. To get a non-vanishing solution the above introduced QUAD approach was used.

In Figure 2a-f) the fiber density found by our approach (FD) is compared to fractional anisotropy (FA) and tract densities (TD) computed by a global fiber tracking approach [5]. While TD is closely correlated to FA the proposed densities are different and do not show the expected [9] picture: FD does not have the maximal dense regions in the Corpus Callosum (CC) and along the cortical spinal tract. The densest regions are around crossing areas and the superior longitudinal fascicle. However note, that the angular maximum map $m a(\boldsymbol{r})=\max _{\boldsymbol{n}} p(\boldsymbol{n}, \boldsymbol{r})=\rho(\boldsymbol{r}) \max _{\boldsymbol{n}} p(\boldsymbol{n} \mid \boldsymbol{r})$ (not shown) resembles much more closely TD or FA, respectively. To get a better impression of the distribution over the whole brain Figure $2 \mathrm{~g}-\mathrm{j}$ ) shows cortical projections of FD. At each point of the white matter isosurface the local average (a neighborhood of 5 voxels) of the fiber density is displayed. While the frontal and parietal lobe show an increased FD, the temporal and occipital lobes appear to be less dense. To get a quantitative picture over the whole group of subjects the WFU pickatlas (http://fmri.wfubmc.edu/software/PickAtlas) was employed to segment in Talairach Daemon Level 2 (lobes) regions. A statistical analysis over the mean FD in the regions is shown in Figure 3, left). For comparison we normalized FD such that the total fiber number $\int \rho$ is the same for all subjects. The density for the different regions is stable over the group and differences between the regions are significant. It is apparent that frontal/parietal regions are more dense than the occipital/temporal regions. Figure 3 shows histograms over the whole white matter area for all 20 subjects. Finally, in Figure 3 (bottom) the CCs of all 20 subjects are shown. The Splenium and Genu appear to have the highest densities while the region around the Isthmus the lowest. There is a small trend of more dense packings towards the anterior part of the $\mathrm{CC}$ body, which seems to be consistent with bound pool imaging [2] and axon density indices [3]. 

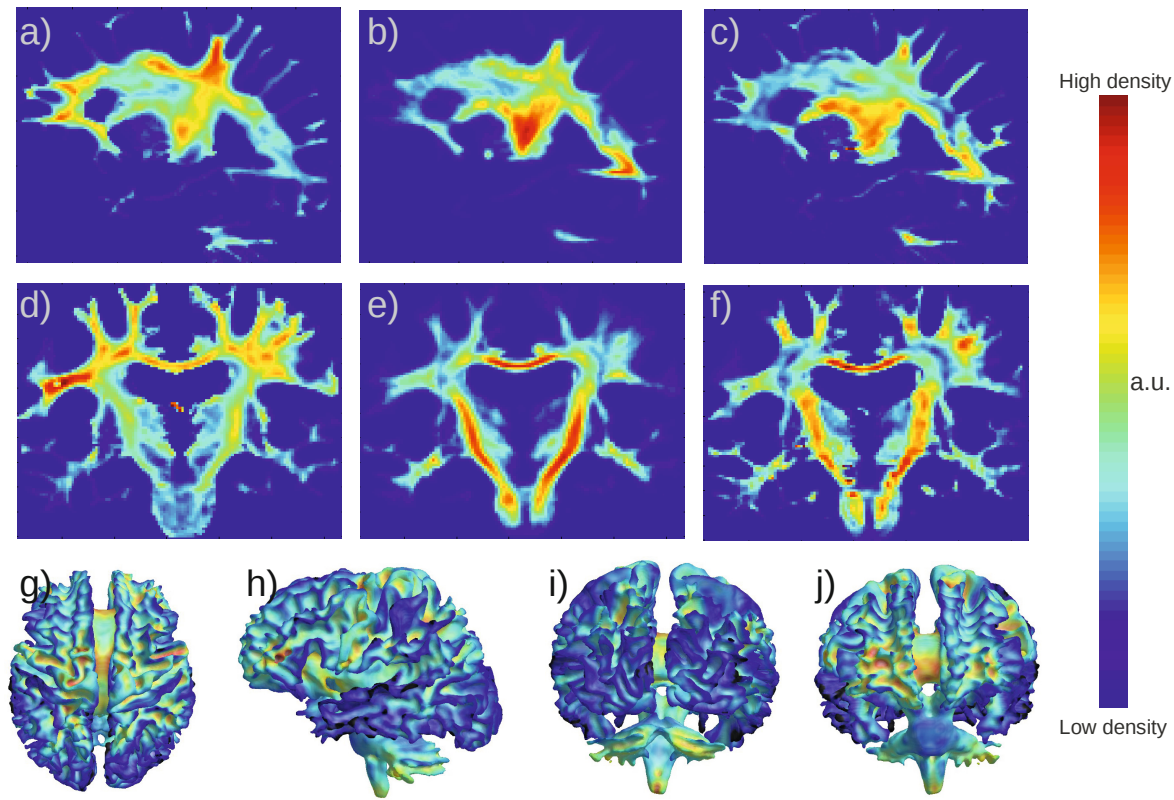

Low density

Fig. 2. a,d) A coronal (at the level of the mid-body of the Corpus Callosum) and saggital section of the fiber densities obtained from the proposed approach. b,e): Tract-densities inferred by global fiber tracking. c,f): Fractional Anisotropy. g-j) Cortical projections of the fiber densities obtained from the proposed approach. The temporal and occipital regions appear to be less populated.

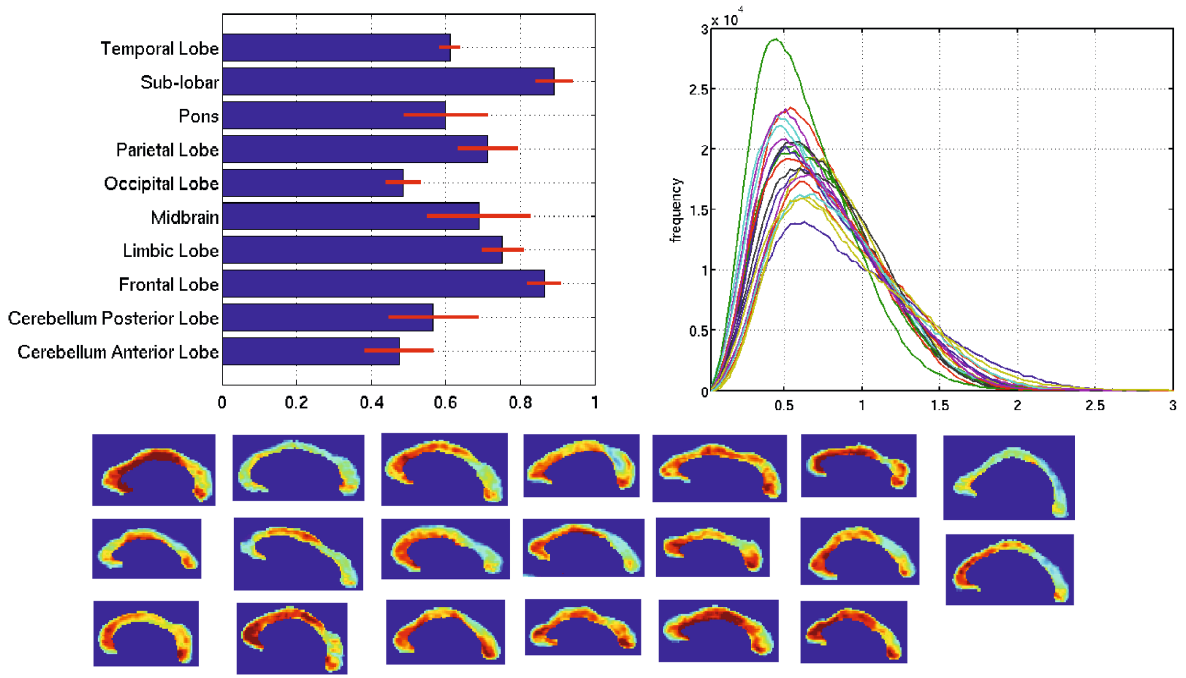

Fig. 3. Top: Statistics over 20 healthy subjects. Top left: mean densities in different regions with group-deviations. Top right: Distribution of densitites over the whole brain of all 20 subjects. Bottom: Fiber densities of the Corpus Callosum for 20 healthy subjects with Genu on the left and Splenium on the right. 


\section{Conclusion}

The main contribution of this work is the proposal of a PDE for tensor-fields, which reflects the fiber conservation law: fibers are not allowed to end somewhere in the white matter. The PDE was implemented with a FEM approach and is solved in a few minutes on a standard PC. Simulations on synthetic data show that the results are quantitative even in the absence of boundary conditions. Although our approach relies on tensor information only, we were able to show that phantoms with a threefold crossing can be resolved. On real DTI data the derived fiber densities appear partially to be consistent with other findings [32], while a rigorous evaluation with histological findings [9] remains challenging.

Acknowledgements. M. Reisert is supported by DFG, grant KI 1089/3-1. H. Skibbe is indebted to the Baden-Württemberg Stiftung for the support of this research project by the Eliteprogramme for Postdocs. Thanks to R. Umarova for providing the data.

\section{References}

1. Jones, D.K. (ed.): Diffusion MRI: Theory, Methods and Applications. Oxford University Press (2010)

2. Stikov, N., Perry, L.M., Mezer, A., Rykhlevskaia, E., Wandell, B.A., Pauly, J.M., Dougherty, R.F.: Bound pool fractions complement diffusion measures to describe white matter micro and macrostructure. Neuroimage 54, 1112-1121 (2011)

3. Alexander, D.C., Hubbard, P.L., Hall, M.G., Moore, E.A., Ptito, M., Parker, G.J., Dyrby, T.B.: Orientationally invariant indices of axon diameter and density from diffusion mri. NeuroImage 52, 1374-1389 (2010)

4. Calamante, F., Tournier, J., Jackson, G., Connelly, A.: Track-density imaging (tdi): superresolution white matter imaging using whole-brain track-density mapping. Neuroimage 53 (2010)

5. Reisert, M., Mader, I., Anastasopoulos, C., Weigel, M., Schnell, S., Kiselev, V.: Global fiber reconstruction becomes practical. Neuroimage 54, 955-962 (2011)

6. Roberts, T., Liu, F., Kassner, A., Mori, S., Guha, A.: AJNR Am. J. Neuroradiol. 26, 2183-2186 (2005)

7. Reisert, M., Kiselev, V.: Fiber continuity: An anisotropic prior for odf estimation. IEEE Trans. Med. Imaging 30, 1274-1283 (2011)

8. Chung, T.: Computational Fluid Dynamics. Cambridge University Press (2002)

9. Burgel, U., Amunts, K., Hoemke, L., Mohlberg, H., Gilsbach, J., Zilles, K.: White matter fiber tracts of the human brain: three-dimensional mapping at microscopic resolution, topography and intersubject variability. Neuroimage 29, 1092-1105 (2006) 